01,07

\title{
Взаимное влияние водорода и вакансий в $\alpha$-цирконии на энергетику их взаимодействия с металлом
}

\author{
(С) Л.А. Святкин ${ }^{1}$, Ю.М. Коротеев ${ }^{1,2}$, И.П. Чернов ${ }^{1}$ \\ ${ }^{1}$ Национальный исследовательский Томский политехнический университет, \\ Томск, Россия \\ ${ }^{2}$ Институт фоизики прочности и материаловедения СО РАН, \\ Томск, Россия \\ E-mail: svyatkin@tpu.ru \\ (Поступила 30 января 2017 г. \\ В окончательной редакции 27 июня 2017 г.)
}

Представлены результаты исследования из первых принципов энергетики взаимодействия водорода и вакансий с $\alpha$-цирконием. Установлено, что наличие вакансий в цирконии увеличивает энергию связи водород-металл, а присутствие водорода в решетке циркония уменьшает энергию образования вакансий. Показано, что водород и вакансии в цирконии образуют комплексы, значительно искажающие решетку металла. Вблизи этих комплексов наблюдается повышение степени ковалентности связей металл-металл и водород-металл.

DOI: 10.21883/FTT.2018.01.45282.020

\section{1. Введение}

Деградация механических свойств конструкционных материалов, обусловленная водородным охрупчиванием [1], стимулирует интенсивные исследования влияния водорода на атомную и электронную структуру металлов и сплавов [2-9]. Исследования твердого раствора ниобий-водород методами электронно-позитронной аннигиляции, дифракции рентгеновского излучения и просвечивающей электронной микроскопии $[3,4]$ показали, что растворение водорода способствует образованию вакансий с последующим формированием сложных комплексов водород-вакансия, структура которых зависит как от концентрации водорода в металле, так и от способа его введения. Теоретические исследования металлов с кубической структурой [5-7], а также ГПУ металлов Ti [8] и $\mathrm{Zr}$ [9] выявили, что наличие водорода и вакансий в решетке металлов приводит к формированию различных комплексов водород-вакансия. Очевидно, что накопление этих комплексов может явиться дополнительным фактором водородного охрупчивания материалов.

Сплавы на основе циркония широко используются для изготовления оболочек топливных элементов ядерных реакторов. В процессе эксплуатации эти элементы конструкции реакторов подвергаются интенсивному воздействию водорода [10] и высоких температур, способствующих образованию равновесных вакансий. Однако к настоящему времени нам известна лишь одна работа, посвященная исследованию комплексов водород-вакансия в цирконии [9]. Поэтому целью настоящего исследования явилось изучение из первых принципов атомной и электронной структуры системы цирконий-водород-вакансия $(\mathrm{Zr}-\mathrm{H}-\mathrm{v})$ при концентрациях водорода и вакансий $~ 6.5$ ат.\%.

\section{2. Метод и детали расчета}

В рамках теории функционала электронной плотности полнопотенциальным методом линеаризованных присоединенных плоских волн [11], реализованном в пакете программ FLEUR [12], проведена оптимизация параметров решетки и релаксация положений всех атомов в расчетной ячейке систем цирконий-водород $(\mathrm{Zr}-\mathrm{H})$, цирконий-вакансия $(\mathrm{Zr}-\mathrm{v})$ и цирконий-водород-вакансия $(\mathrm{Zr}-\mathrm{H}-\mathrm{v})$. Обменно-корреляционные эффекты учитывались с использованием обобщенного градиентного приближения в форме Пердью-БуркеЕрнцерхофа (PBE) [13]. Радиусы МТ-сфер атомов Zr и Н были выбраны равными 1.058 и $0.529 \AA$ соответственно, что позволяло атомам водорода свободно помещаться в междоузлиях кристаллической решетки $\alpha-Z r$. Самосогласование электронной плотности считалось достигнутым, когда среднеквадратичное отклонение между входной и выходной зарядовыми плотностями валентных электронов становилось меньше $10^{-3}$ электронов $/ \AA^{3}$, что соответствовало сходимости полной энергии не хуже $0.02 \mathrm{meV}$. Релаксация считалась завершенной, когда силы, действующие на каждый атом расчетной ячейки, становились меныше $50 \mathrm{meV} / \AA$.

Расчетные ячейки всех рассмотренных систем представляли собой блок элементарных ячеек ГПУ решетки циркония $2 \times 2 \times 2$ (рис. 1). Для удобства обсуждения результатов атомы циркония на рисунках пронумерованы. Расчетная ячейка твердого раствора $\mathrm{Zr}-\mathrm{H}$ содержала 16 атомов $\mathrm{Zr}$ и один атом Н в тетраэдрическом Т- или октаэдрическом О-междоузлии (рис. 1,a). В случае циркония с вакансиями $(\mathrm{Zr}-\mathrm{v})$ (рис. $1, b)$ один из узлов (12) решетки $v$ был вакантным, так что расчетная ячейка содержала 15 атомов $\mathrm{Zr}$. Для твердого раствора с вакансиями $(\mathrm{Zr}-\mathrm{H}-\mathrm{v})$ было рассмотрено 

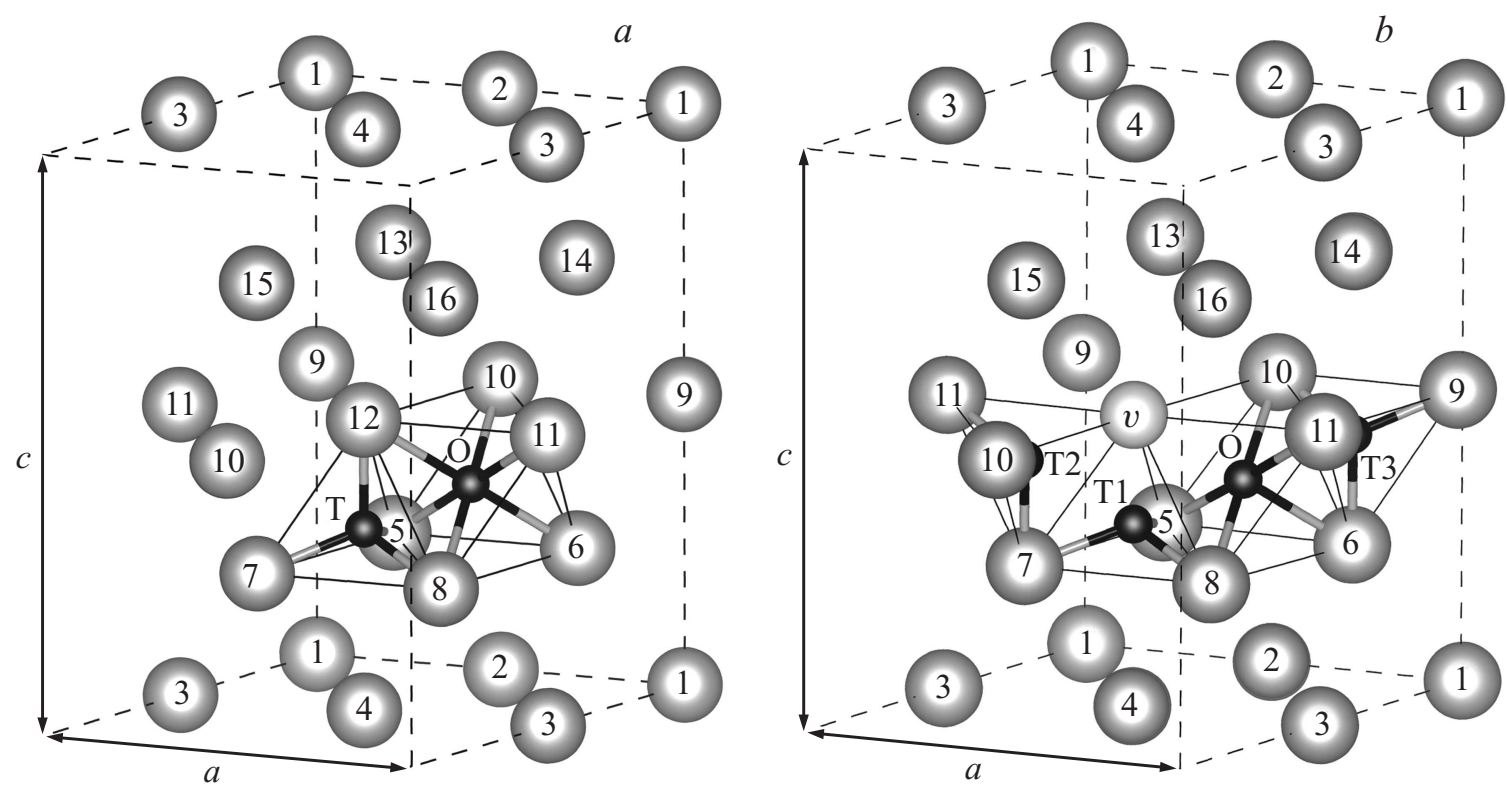

Рис. 1. Расчетные ячейки систем $\mathrm{Zr}-\mathrm{H}(a)$ и $\mathrm{Zr}-\mathrm{H}-v(b)$, где атомы водорода занимают тетраэдрические (T, Т1, Т2 и Т3) или октаэдрические $(\mathrm{O})$ междоузлия; $a$ и $c$ - параметры расчетной ячейки.

пять вариантов размещения водорода в решетке металла: в вакансии $v$ и вблизи вакансии в тетраэдрических Т1, Т2 и Т3 и октаэдрическом О-междоузлиях (рис. 1,b). Рассмотренные тетраэдрические междоузлия, как видно из рис. $1, b$, отличаются друг от друга своим положением относительно вакансии. Для каждой системы было рассчитано распределение валентной зарядовой плотности.

\section{3. Результаты и обсуждение}

3.1. Оптимизация и релаксация атомной структуры. Параметры решетки всех рассмотренных систем приведены в табл. 1 и 2. Значения, полученные нами для чистого $\mathrm{Zr}$ и системы $\mathrm{Zr}-\mathrm{H}$ (табл. 1), хорошо согласуется как с результатами экспериментов [14,17], так и расчетов других авторов $[15,16]$. При тетраэдрической координации водорода параметры решетки циркония $a$ и $c$ увеличиваются соответственно на 0.19 и 0.80\% по сравнению с чистым металлом, а при октаэдрической - увеличивается на $0.40 \%$ лишь параметр $c$. Образование вакансии вызывает сокращение объема кристалла, уменьшая параметры $a$ и $c$ соответственно на 0.50 и $1.70 \%$.

Растворение водорода в системе $\mathrm{Zr}-v$ может приводить как к увеличению, так и к уменьшению параметров решетки в зависимости от занимаемого им междоузлия (табл. 2). Так при размещении водорода в тетраэдрических междоузлиях Т1 и Т2, ближайших к вакансии, наблюдается увеличение параметра $a$ на 0.50 и $0.22 \%$ и уменьшение параметра $c$ на 0.26 и $0.16 \%$ соответственно. Размещение водорода в ближайшем к вакансии октаэдрическом междоузлии и удаленном от нее на расстояние $\sim a$ тетраэдрическом междоузлии Т3 приводит к увеличению параметров решетки практически на ту же величину, как и в бездефектном металле. В случае, когда атом водорода находится в центре вакансии, параметр $a$ практически не изменяется, а параметр $c$ уменьшается на $0.75 \%$.

Важность учета в нашем исследовании релаксации атомной структуры демонстрирует система $\mathrm{Zr}-\mathrm{H}$. Так в идеальной ГПУ-решетке циркония (т. е. без учета релаксации) энергетически наиболее выгодным (на $30 \mathrm{meV}$ ) положением водорода является октаэдрическое междоузлие. В результате релаксации происходят небольшие смещения ближайших к водороду атомов металла

Таблица 1. Параметры решетки чистого $\mathrm{Zr}$ и систем $\mathrm{Zr}-v$, $\mathrm{Zr}-\mathrm{H}$

\begin{tabular}{c|c|l|l}
\hline \multirow{2}{*}{ Система } & \multicolumn{3}{|c}{ Параметры решетки, } \\
\cline { 2 - 4 } & Настоящая работа & Другие расчеты & Эксперимент \\
\hline $\mathrm{Zr}$ & & $a=3.213[15]$ & \\
& $a=3.235$ & $c=5.157[15]$ & $a=3.230[14]$ \\
& $c=5.157$ & $a=3.23[16]$ & $c=5.145[14]$ \\
& & $c=5.18[16]$ & \\
\hline $\mathrm{Zr}-\mathrm{H}^{\mathrm{T}}$ & $a=3.241$ & $a=3.246[15]$ & \\
& $c=5.198$ & $c=5.233[15]$ & $a=3.242[17]$ \\
\hline $\mathrm{Zr}-\mathrm{H}^{\mathrm{O}}$ & $a=3.235$ & $a=3.244[15]$ & $c=5.174[17]$ \\
& $c=5.178$ & $c=5.203[15]$ & \\
\hline $\mathrm{Zr}-v$ & $a=3.219$ & - & \\
& $c=5.067$ & &
\end{tabular}


Таблица 2. Параметры решетки системы $\mathrm{Zr}-\mathrm{H}-\mathrm{v}$

\begin{tabular}{c|c|c|c|c|c}
\hline Система & $\mathrm{Zr}-\mathrm{H}^{v}$ & $\mathrm{Zr}-\mathrm{H}^{\mathrm{T} 1}-\mathrm{v}$ & $\mathrm{Zr}-\mathrm{H}^{\mathrm{T} 2}-\mathrm{v}$ & $\mathrm{Zr}-\mathrm{H}^{\mathrm{T} 3}-\mathrm{v}$ & $\mathrm{Zr}-\mathrm{H}^{\mathrm{O}}-\mathrm{v}$ \\
\hline Параметры & $a=3.22$ & $a=3.235$ & $a=3.226$ & $a=3.227$ & $a=3.218$ \\
решетки, $\AA$ & $c=5.029$ & $c=5.054$ & $c=5.059$ & $c=5.115$ & $c=5.091$
\end{tabular}

$(\sim 0.04-0.09 \AA)$ и энергетически наиболее выгодным (на $34 \mathrm{meV}$ ) положением водорода становится тетраэдрическое междоузлие. Это хорошо согласуется с результатами эксперимента [18] и других теоретических работ $[15,16]$. Как показали наши расчеты, атом водорода в обоих междоузлиях при релаксации практически не смещается из центра соответствующего полиэдра. В случае тетраэдрической координации заметное смещение испытывают ближайшие к водороду атомы циркония в вершинах тетраэдра (рис. 2,a): атом 12 сдвигается на $0.085 \AA$, а атомы 5,7 и 8 в основании тетраэдра на $0.044 \AA$. Вместе с этим наблюдается смещение на $0.034 \AA$ атома 4 в вершине соседнего тетраэдра с тем же основанием. При октаэдрической координации водорода атомы циркония испытывают меньшие смещения, но количество смещающихся атомов больше (рис. 2, $b$ ). Атомы 5, 6, 8 и 10, 11, 12 в вершинах октаэдра сдвигаются преимущественно в базальной плоскости на $0.038 \AA$, удаляясь от атома водорода в направлении ближайшей ГПУ-пустоты.

Образование вакансии вызывает заметное искажение кристаллической решетки металла (рис. 2,c), как в слое, где она находится, так и в соседних слоях. Атомы 9, 10 и 11 в вершинах шестиугольника, в центре которого находится вакансия, сдвигаются в направлении ближайшей ГПУ-пустоты на $0.078 \AA \AA$. Атомы 5,7 и 8, а также 13,15 и 16, находящиеся в соседних слоях, приближаются к вакансии на $0.052 \AA$, смещаясь преимущественно вдоль гексагональной оси.
Важность учета релаксационных эффектов демонстрирует также и система $\mathrm{Zr}-\mathrm{H}-v$. Расчеты ее полной энергии без учета релаксации решетки металла показали, что наиболее энергетически выгодным положением водорода является ближайшее к вакансии октаэдрическое междоузлие, которое на $28 \mathrm{meV}$ выгоднее тетраэдрического междоузлия Т2 и более чем на $115 \mathrm{meV}$ - междоузлий Т1 и Т3. В результате релаксации наиболее предпочтительным положением водорода становится междоузлие $\mathrm{T} 2$, где полная энергия системы $\mathrm{Zr}-\mathrm{H}-\mathrm{v}$ на $70 \mathrm{meV}$ меньше, чем при октаэдрической координации.

Наличие в системе $\mathrm{Zr}-\mathrm{H}-v$ обоих типов дефектов (растворенного водорода и вакансий) приводит к увеличению степени релаксации решетки металла. В системе $\mathrm{Zr}-\mathrm{H}^{\mathrm{T} 1}-v$ (рис. $3, a$ ) большая часть атомов циркония, участвующих в релаксации, смещается подобно тому, как это происходит в системе $\mathrm{Zr}-v$. Присутствие водорода проявляется в изменении направления и величины смещения ближайших к нему атомов металла 5, 7 и 8, отклоняя его в сторону вакансии и увеличивая на $70 \%$. Кроме того, водород вызывает сдвиг атома 4, как и в системе $\mathrm{Zr}-\mathrm{H}^{\mathrm{T}}$ (рис. 2, $a$ ), однако этот сдвиг почти в 2 раза больше $(0.083 \AA)$. В свою очередь, влияние вакансии на положение атома водорода выражается в его заметном сдвиге (на $0.264 \AA$ ) из центра тетраэдра в направлении вакансии.

Наибольшее влияние вакансий и водорода на релаксацию решетки металла наблюдается в системе $\mathrm{Zr}-\mathrm{H}^{\mathrm{T} 2}-v$ (рис. $3, b)$. В этом случае атом водорода в результате

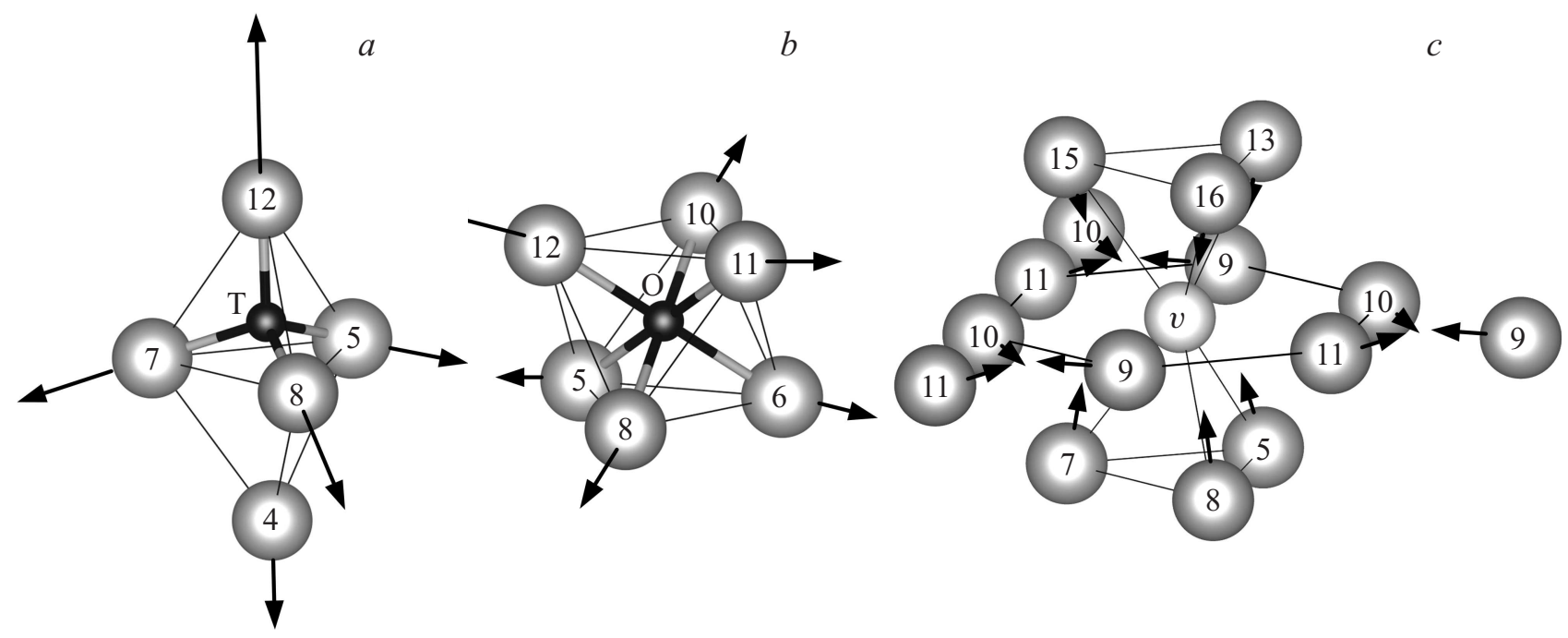

Рис. 2. Схема релаксации решетки циркония вблизи атома водорода в тетраэдрическом $(a)$ и октаэдрическом $(b)$ междоузлиях системы $\mathrm{Zr}-\mathrm{H}$ и вблизи вакансии в системе $\mathrm{Zr}-\mathrm{v}$. 
$a$

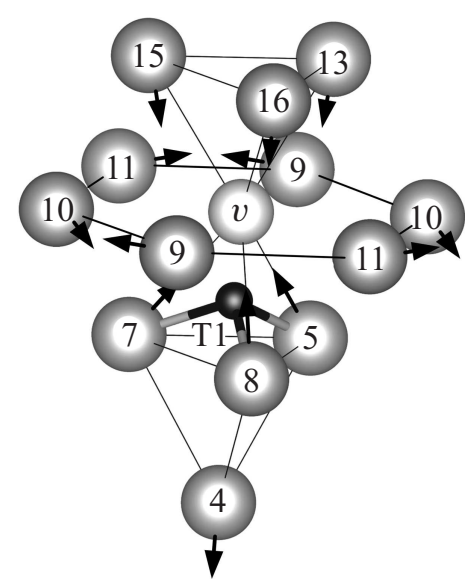

$b$

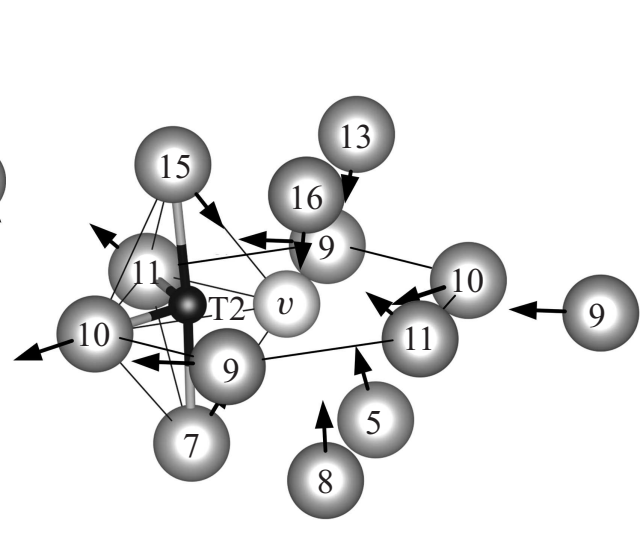

$c$

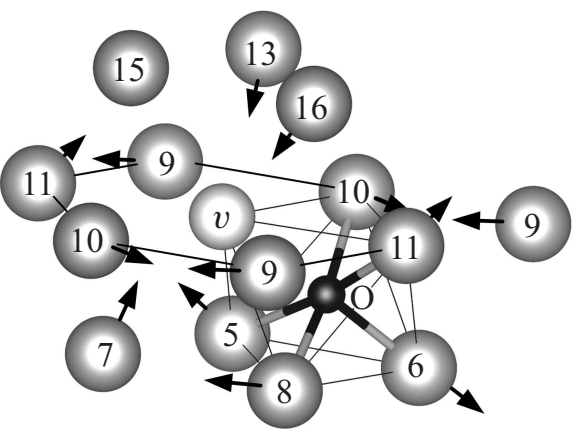

Pис. 3. Схема релаксации решетки циркония вблизи комплекса водород-вакансия при тетраэдрической Т1 (a), Т2 $(b)$ и октаэдрической $\mathrm{O}(c)$ координациях атома $\mathrm{H}$.

релаксации смещается из центра тетраэдра на $0.632 \AA$ вдоль гексагональной оси и попадает в базальную плоскость, в которой сдвигается в направлении вакансии на $0.159 \AA$ А. При этом в базальной плоскости ближайшие к водороду атомы циркония 10 и 11 смещаются от него на $0.088 \AA$, но не в ГПУ-пустоту, как в случае $\mathrm{Zr}-v$, а в ГЦК-пустоту, как в системе $\mathrm{Zr}-\mathrm{H}^{\mathrm{T}}$. Это приводит к увеличению смещения атома 9, лежащего в этой же плоскости, в 2 раза и уменьшению сдвигов атомов 5,8 и 13, 16 в соседних слоях в сторону вакансии почти в 1.5 раза по сравнению с системой $\mathrm{Zr}-v$. Значительное влияние водорода испытывают атомы 15 и 7, лежащие над и под ним в соседних слоях: в отличие от системы $\mathrm{Zr}-v$, где эти атомы смещаются преимущественно вдоль гексагональной оси, их сдвиг происходит в направлении вакансии и увеличивается в 2.25 раза. Это, в свою очередь, приводит к смещению атомов 2, 3 и 4 (см. рис. $1, b$, т. к. на рис. $3, b$ они не показаны) в направлении ближайшей ГЦК-пустоты до $0.06 \AA$.

При расположении водорода в тетраэдрическом междоузлии Т3 смещение атомов металла вокруг вакансии уменьшается в 1.5-2 раза по сравнению с тем, что наблюдается в системе $\mathrm{Zr}-v$ (рис. 2,c). Влияние вакансии на релаксацию решетки металла вблизи водорода проявляется в усилении смещений атомов циркония 14 (на $30 \%$ ) и 6 (на 60\%), находящихся над и под атомом водорода (рис. $1, b)$ и соответствующих атомам 4 и 12 в системе $\mathrm{Zr}-\mathrm{H}^{\mathrm{T}}$ (рис. 2, a). Наблюдается также смещение атома водорода вдоль гексагональной оси из центра тетраэдра к его вершине (атому 6, рис. $1, b$ ) на $0.034 \AA$.

Совместное влияние вакансий и водорода на релаксацию решетки металла в системе $\mathrm{Zr}-\mathrm{H}^{\mathrm{O}}-\mathrm{v}$ (рис. 3, c) проявляется в уменьшении по сравнению с системой $\mathrm{Zr}-v$ почти на треть смещений атомов 10 и 11 в базальной плоскости и их сдвиге на $0.02 \AA$ вдоль гексагональной оси. При этом на четверть увеличивается смещение атома 9. Присутствие водорода усиливает также смещения атомов 7 (почти в 2 раза), а также 13 и 16 (в 1.3 раз) и значительно уменьшает смещения атомов 5, 8 и 15. Наличие вакансии в вершине октаэдра, в котором располагается водород, увеличивает смещение атома 6 на 10\%, дополнительно сдвигая его вдоль гексагональной оси на $0.03 \AA$. Это вызывает смещение атома 1 на $0.062 \AA$ от атома 6 (см. рис. $1, b$, так как на рис. 3, этот атом не показан), которое более чем в 2 раза превышает смещения этого атома в системах $\mathrm{Zr}-\mathrm{H}^{\mathrm{O}}$ и $\mathrm{Zr}-v$. Влияние вакансии проявляется также в значительном смещении $(0.134 \AA)$ атома водорода из центра октаэдра вдоль его оси симметрии, проходящей через вакантный узел, в направлении атома 6 (рис. 3,c). Заметим, что при тетраэдрической координации Т1 и Т2 атом водорода в результате релаксации сдвигается в направлении вакансии.

В случае, когда атом водорода размещался в вакансии (система $\mathrm{Zr}-\mathrm{H}^{v}$ ), даже небольшие его смещения из центра вакансии приводили к возникновению сил, сдвигающих атом Н в ближайшее междоузлие, что свидетельствует о неустойчивости этой конфигурации комплекса водород-вакансия в цирконии.

3.2. Энергетика системы $\mathrm{Zr}-\mathrm{H}-v$. Для исследования взаимного влияния водорода и вакансий на энергетику их взаимодействия с цирконием были рассчитаны энергия связи водорода $E_{\mathrm{H}} \mathrm{c} \mathrm{Zr}$ и системой $\mathrm{Zr}-\mathrm{v}$

$$
E_{\mathrm{H}}=E\left(\mathrm{Zr}_{N-x}\right)+\frac{1}{2} E\left(\mathrm{H}_{2}\right)-E\left(\mathrm{Zr}_{N-x} \mathrm{H}\right)
$$

энергия образования вакансии $E_{v}$ в $\mathrm{Zr}$ и системе $\mathrm{Zr}-\mathrm{H}$

$$
\begin{gathered}
E_{v}=E\left(\mathrm{Zr}_{N-x}\right)-\frac{N-x}{N} E\left(\mathrm{Zr}_{N}\right), \\
E_{v}=E\left(\mathrm{Zr}_{N-x} \mathrm{H}\right)+\frac{x}{N} E\left(Z_{N}\right)-E\left(Z r_{N} \mathrm{H}\right)
\end{gathered}
$$


Таблица 3. Энергия связи водорода в цирконии $E_{\mathrm{H}}[\mathrm{eV}]$

\begin{tabular}{|c|c|c|c|c|c|c|c|}
\hline Система & $\mathrm{Zr}-\mathrm{H}^{\mathrm{T}}$ & $\mathrm{Zr}-\mathrm{H}^{\mathrm{O}}$ & $\mathrm{Zr}-\mathrm{H}^{v}$ & $\mathrm{Zr}-\mathrm{H}^{\mathrm{T} 1}-v$ & $\mathrm{Zr}-\mathrm{H}^{\mathrm{T} 2}-\mathrm{v}$ & $\mathrm{Zr}-\mathrm{H}^{\mathrm{T} 3}-\mathrm{v}$ & $\mathrm{Zr}-\mathrm{H}^{\mathrm{O}}-\mathrm{v}$ \\
\hline Настоящая работа & 0.426 & 0.392 & -0.603 & 0.522 & 0.671 & 0.512 & 0.601 \\
\hline Другие расчеты & $\begin{array}{l}0.609[9] \\
0.45[15]\end{array}$ & $\begin{array}{l}0.549[9] \\
0.35[15]\end{array}$ & - & - & - & - & - \\
\hline Эксперимент & $\begin{array}{l}0.3[18] \\
0.66[19]\end{array}$ & - & - & - & - & - & - \\
\hline
\end{tabular}

Таблица 4. Энергия образования вакансии $E_{v}[\mathrm{eV}]$ в $\mathrm{Zr}$ и в системе $\mathrm{Zr}-\mathrm{H}$

\begin{tabular}{l|c|c|c|c|c}
\hline Система & $\mathrm{Zr}-v$ & $\mathrm{Zr}-\mathrm{H}^{\mathrm{T} 1}-v$ & $\mathrm{Zr}-\mathrm{H}^{\mathrm{T} 2}-v$ & $\mathrm{Zr}-\mathrm{H}^{\mathrm{T} 3}-v$ & $\mathrm{Zr}-\mathrm{H}^{\mathrm{O}}-v$ \\
\hline Настоящая работа & 2.096 & 1.960 & 1.811 & 2.010 & 1.887 \\
Другие расчеты & $2.04[20]$ & - & - & - & - \\
Эксперимент & $\geq 1.5[21,22]$ & - & - & - & -
\end{tabular}

Таблица 5. Энергия связи водорода с вакансией $E_{\mathrm{H}-v}[\mathrm{eV}]$ в цирконии

\begin{tabular}{c|c|c|c|c|c}
\hline Система & $\mathrm{Zr}-\mathrm{H}^{v}$ & $\mathrm{Zr}-\mathrm{H}^{\mathrm{T} 1}-v$ & $\mathrm{Zr}-\mathrm{H}^{\mathrm{T} 2}-v$ & $\mathrm{Zr}-\mathrm{H}^{\mathrm{T} 3}-v$ & $\mathrm{Zr}-\mathrm{H}^{\mathrm{O}}-\mathrm{v}$ \\
\hline Настоящая работа & -1.029 & 0.096 & 0.244 & 0.086 & 0.205 \\
Другие расчеты & $-1.16[9]$ & $0.08[9]$ & $0.22[9]$ & - & $0.18[9]$
\end{tabular}

и энергия связи водорода с вакансией $E_{\mathrm{H}-\nu}$ в цирконии

$$
E_{\mathrm{H}-v}=E\left(\mathrm{Zr}_{N} \mathrm{H}\right)+E\left(\mathrm{Zr}_{N-x}\right)-E\left(\mathrm{Zr}_{N}\right)-E\left(\mathrm{Zr}_{N-x} \mathrm{H}\right),
$$

где $E\left(\mathrm{Zr}_{N}\right), E\left(\mathrm{Zr}_{N} \mathrm{H}\right), E\left(\mathrm{Zr}_{N-x}\right)$ и $E\left(\mathrm{Zr}_{N-x} \mathrm{H}\right)$ - полные энергии соответственно чистого $\alpha-\mathrm{Zr}$ и систем $\mathrm{Zr}-\mathrm{H}, \mathrm{Zr}-v$ и $\mathrm{Zr}-\mathrm{H}-v$ с концентрацией вакансий $x / N$ $(x$ и $N-$ количество вакансий и узлов решетки в расчетной ячейке соответственно), $E\left(\mathrm{H}_{2}\right)$ - энергия связи молекулы водорода.

Результаты расчета энергии связи водорода с $\mathrm{Zr}$ и системой $\mathrm{Zr}-v$ представлены в табл. 3. Видно, что энергия связи водорода в бездефектном цирконии имеет положительные значения, что свидетельствует о формировании связи между атомами водорода и циркония. Наибольшая энергия связи соответствует тетраэдрической координации атома водорода. Это подтверждается результатами эксперимента по дифракции нейтронов в системе $\mathrm{Zr}-\mathrm{H}$ [18], показавшего, что при комнатной температуре водород в цирконии занимает тетраэдрические междоузлия. Следует отметить, что небольшое различие вычисленных нами энергий связи водорода в октаэдрическом и тетраэдрическом междоузлиях $\left(\Delta E_{\mathrm{H}} \sim 34 \mathrm{meV}\right)$ позволяет ожидать, что уже при температуре выше $120^{\circ} \mathrm{C}$, а тем более при температурах работы ядерного реактора $\left(350-400^{\circ} \mathrm{C}\right)$, атомы водорода почти с равной вероятностью будут занимать оба междоузлия. Рассчитанные в настоящей работе значения количественно достаточно хорошо согласуются с результатами псевдопотенциальных расчетов [15] и качественно - с результатами расчетов [9], превышающих наши значения на величину 40\%.
Экспериментальные значения энергии связи $E_{\mathrm{H}}$ по разным источникам составляют $0.3 \mathrm{eV}$ [18] и $0.66 \mathrm{eV}$ [19], так что полученные нами результаты находятся между этими значениями.

Из табл. 3 также видно, что наличие вакансий в металле увеличивает энергию связи водорода с цирконием на 23, 58 и 20\% в тетраэдрических Т1, Т2 и Т3 соответственно и на $53 \%$ в октаэдрическом междоузлиях по сравнению с бездефектным кристаллом. Энергия связи циркония с водородом вблизи вакансии (междоузлия Т1, Т2 и О) больше, чем вдали от нее (междоузлие Т3). При этом наибольшее значение $E_{\mathrm{H}}$ соответствует размещению атома водорода в тетраэдрическом междоузлии Т2. Энергия связи циркония с водородом, находящимся в вакансии, отрицательна $(-0.603 \mathrm{eV})$, то есть для размещения атома водорода в вакансии необходимо затратить энергию.

Энергия образования вакансии в $\mathrm{Zr}$ и системе $\mathrm{Zr}-\mathrm{H}$ представлена в табл. 4. Рассчитанное нами значение $E_{v}$ в $\mathrm{Zr}$ хорошо согласуется с результатами теоретических исследований [20], и вполне разумно - с данными экспериментов [21,22]. Присутствие водорода в цирконии понижает эту энергию для ближайших к нему атомов металла на величину порядка $0.14-0.29 \mathrm{eV}$. Для более удаленных атомов $\mathrm{Zr}$ это изменение не столь велико $(0.086 \mathrm{eV})$. Это свидетельствует о том, что водород заметно ослабляет связь между ближайшими к нему атомами циркония. Аналогичные результаты были получены в псевдопотенциальных расчетах [23] для ГЦК металлов, где образование гидридов понижает энергию образования вакансий на $0.8-4.2 \mathrm{eV}$. 

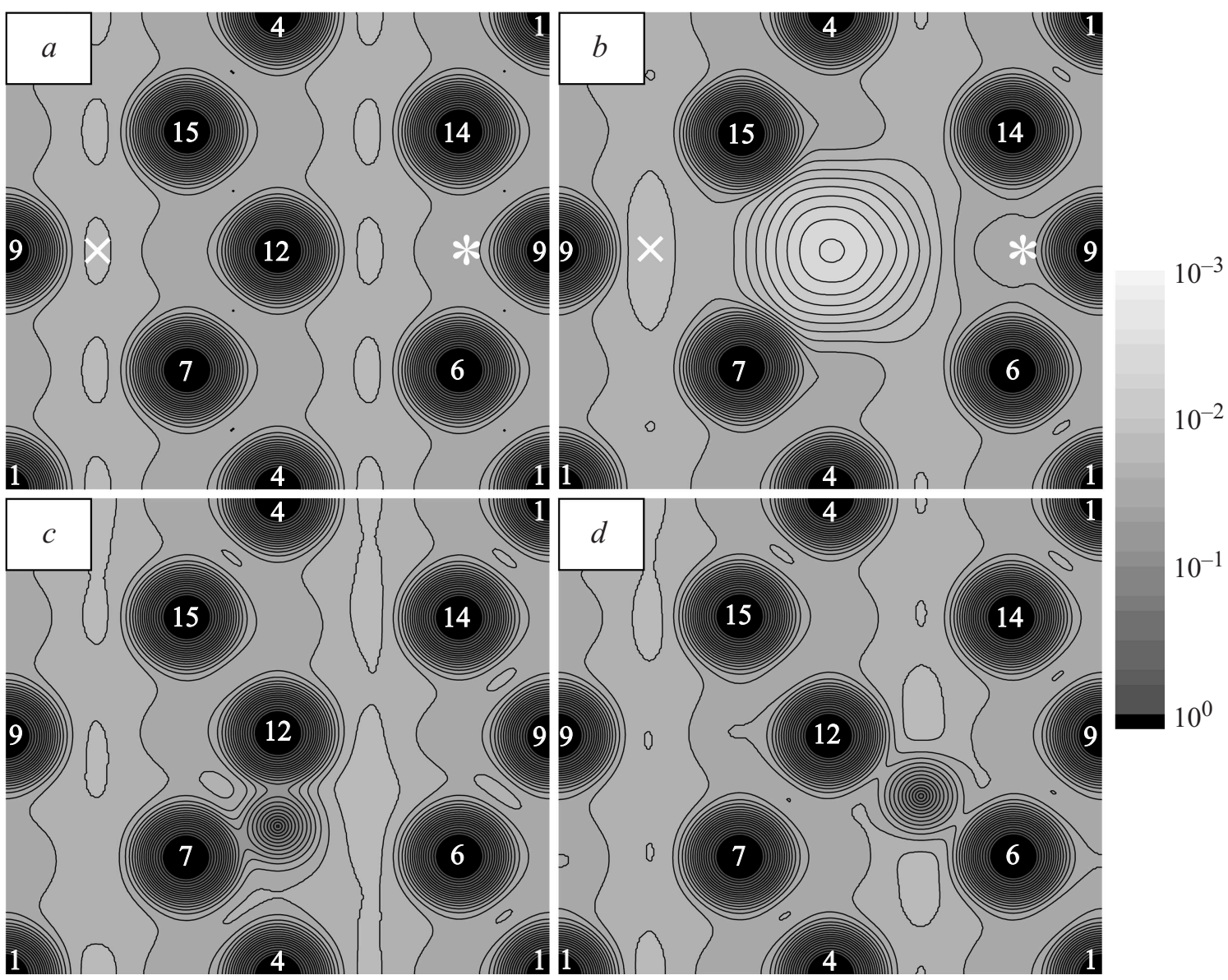

Рис. 4. Распределение электронной плотности чистого $\mathrm{Zr}(a)$ и систем $\mathrm{Zr}-v(b), \mathrm{Zr}-\mathrm{H}^{\mathrm{T}}(c)$ и $\mathrm{Zr}-\mathrm{H}^{\mathrm{O}}(d)$ в плоскости $(11 \overline{2} 0)$, проходящей через вакансии, атомы циркония и водорода. Шкала градаций цвета приведена в единицах electron/Bohr ${ }^{3}$.

В табл. 5 представлены результаты расчетов энергии связи водорода с вакансией в цирконии. Отрицательное значение этой величины в системе $\mathrm{Zr}-\mathrm{H}^{v}$ свидетельствует о неустойчивом положении водорода в вакансии, что уже отмечалось при обсуждении эффектов релаксации, и согласуется с результатами расчетов для Ті [8] и $\mathrm{Zr}$ [9]. В случае расположения водорода в междоузлиях величина $E_{\mathrm{H}-v}$ варьируется от 0.086 до $0.244 \mathrm{eV}$. Наибольшее значение энергии связи достигается при размещении атома Н в тетраэдрическом междоузлии Т2. Как следует из табл. 5, полученные нами значения хорошо согласуются результатами псевдопотенциальных расчетов [9].

3.3. Распределение электронной плотности. С целью выявления влияния вакансий и атомов водорода на взаимодействие между атомами циркония был проведен сравнительный анализ распределений электронной плотности чистого металла, металла с вакансиями, а также систем $\mathrm{Zr}-\mathrm{H}$ и $\mathrm{Zr}-\mathrm{H}-v$, представленных на рис. 4-6. Видно, что образование вакансий приводит к значительному перераспределению электронной плотности металла (рис. $4, a-b$ и $5, a-b$ ), обусловленному уменьшением числа валентных электронов в системе за счет удаления атома циркония из узла решетки и связанной с этим разгибридизацией части валентных состояний металла. Валентный заряд в МТ-сферах циркония возрастает по сравнению с бездефектным металлом на величину, не превышающую $0.020 e$. Максимальное увеличение заряда $(0.020 e)$ наблюдается в МТ-сферах атомов циркония 9, 10 и 11, окружающих вакансию в базальной плоскости (рис. 5, $b$ ). В этой плоскости также происходит значительный перенос заряда из области ГЦК-пустот $(\times)$ решетки циркония в область ГПУ-пустот $(*)$, что наиболее отчетливо видно при сравнении рис. 4, $a$ и 4, $b$. Это вызывает ослабление взаимодействия между указанными выше атомами $\mathrm{Zr}$ в области ГЦК-пустот и усиление их взаимодействия в области ГПУ-пустот, проявляющегося в появлении дополнительной изолинии, охватывающей эти атомы (рис. 5, $b$ ). Валентный заряд в МТ-сферах атомов 14 и 6, находящихся в соседних базальных плоскостях над и под этими ГПУ-пустотами (рис. 4, $a$ и $b$ ), увеличивается меньше (на 0.011e). В МТ-сферах атомов, максимально удаленных от вакансии (атомы 1,2 и 3, рис 1,b), увеличение валентного заряда составляет $\sim 0.008 e$ и 


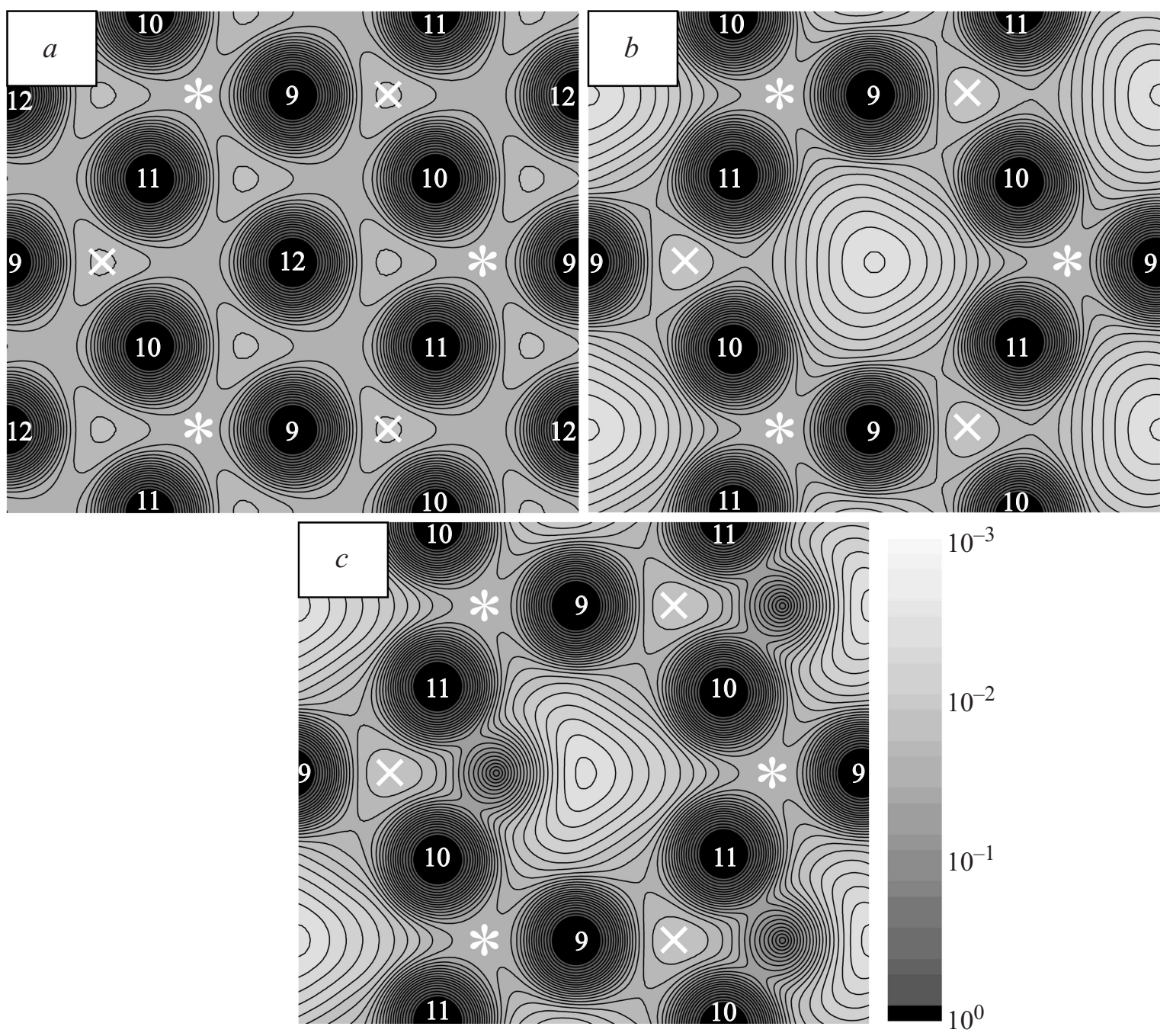

Рис. 5. Распределение электронной плотности чистого $\mathrm{Zr}(a)$ и систем $\mathrm{Zr}-v(b)$ и $\mathrm{Zr}-\mathrm{H}^{\mathrm{T} 2}-v(c)$ в плоскости $(0001)$, проходящей через вакансии и атомы циркония. Шкала градаций цвета приведена в единицах electron/Bohr³.

обусловлено, как показал анализ, уменьшением объема кристалла за счет образования вакансии.

Незначительное увеличение заряда $\sim 0.003 e$ имеет место в МТ-сферах атомов 13, 15, 16 и 5, 7, 8, находящихся соответственно над и под вакансией в соседних базальных плоскостях (рис. $1, b, 4, a$ и $b$ ). Вместе с тем, в ГПУ, и ГЦК-пустотах вблизи этих атомов электронная плотность заметно повышается (на $\sim 2$ и $10 \%$ соответственно), что указывает на усиление взаимодействия между ними за счет регибридизации части валентных состояний металла. Кроме того, образование вакансий приводит к ослаблению связи между парой атомов 7-15 и однотипными парами 5-13 и 8-16 (рис. $1, b$ ), о чем свидетельствует разрыв общего контура изолиний распределения электронной плотности (рис. $4, b$ ), охватывающего эти атомы в чистом металле (рис. 4, $a$ ). В MT-сфере атома 4 и ближайших к нему междоузлиях заметных изменений валентной электронной плотности не наблюдается.
Растворяясь в решетке циркония, водород вызывает заметное перераспределение электронной плотности металла, что отчетливо видно на рис. $4, a, c$ и $d$. В частности рис. $4, c$ и $d$ демонстрируют, что между атомами $\mathrm{H}$ и ближайшими к нему атомами $\mathrm{Zr}$ наблюдается повышенный уровень электронной плотности: эти атомы охвачены общими изолиниями электронной плотности. Это свидетельствует о формировании связи металл-водород, заметная доля которой приходится на ковалентную составляющую. При этом в тетраэдрическом междоузлии атом водорода сильнее связывается с атомами циркония, чем в октаэдрическом, так как охватываются большим количеством изолиний. Это коррелирует с результатами расчета энергии связи водорода в цирконии: $E_{\mathrm{H}}(\mathrm{T})>E_{\mathrm{H}}(\mathrm{O})$ (табл. 3).

Распределение электронной плотности системы $\mathrm{Zr}-\mathrm{H}-\mathrm{v}$ показано на рис. $5, c$ и $6, a-c$. Видно, что присутствие вакансий усиливает ковалентную составляющую связи между атомами водорода и циркония, о чем 

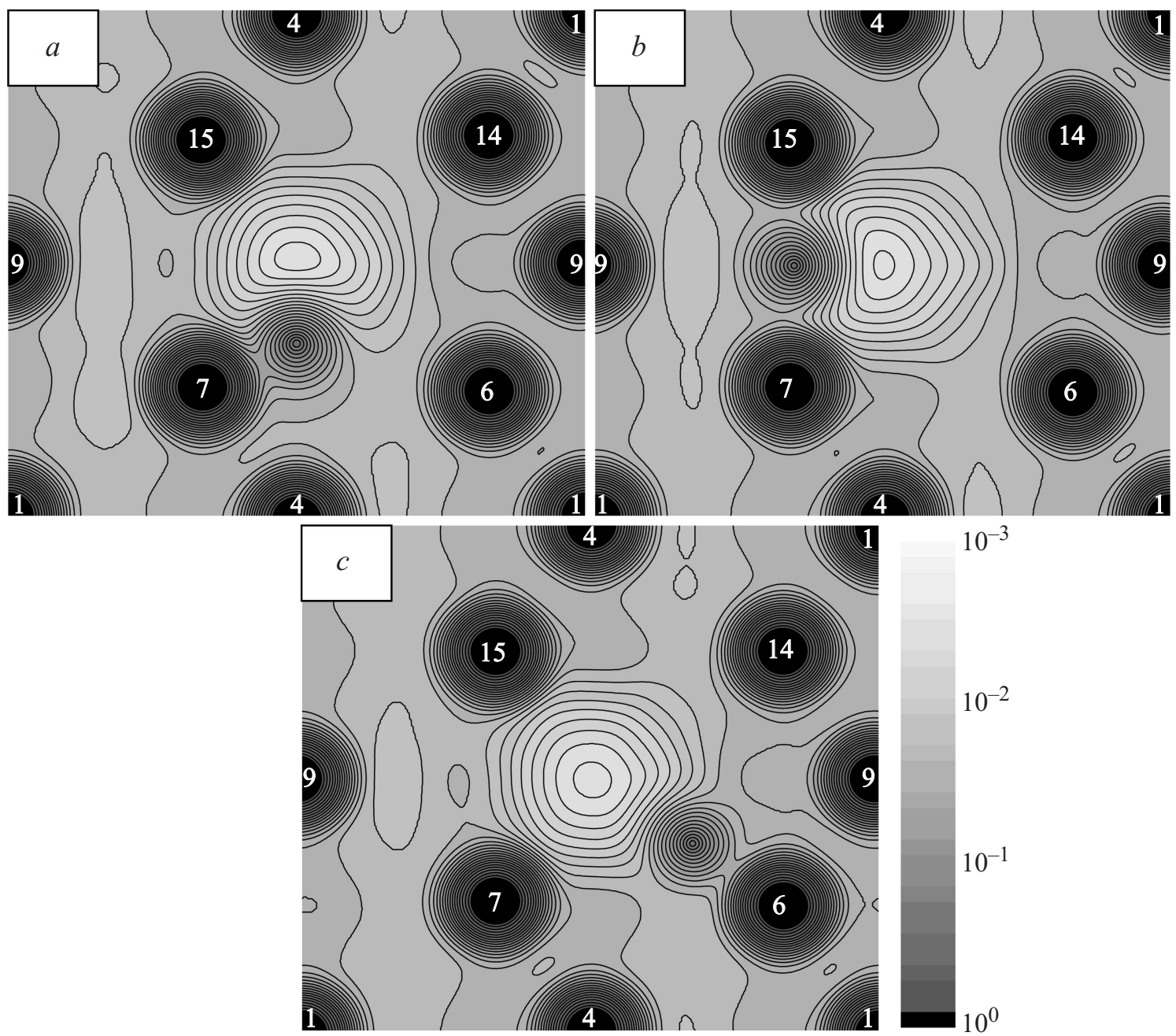

Рис. 6. Распределение электронной плотности систем $\mathrm{Zr}-\mathrm{H}^{\mathrm{T} 1}-v(a), \mathrm{Zr}-\mathrm{H}^{\mathrm{T} 2}-v(b)$ и $\mathrm{Zr}-\mathrm{H}^{\mathrm{O}}-v(c)$ в плоскости $(11 \overline{2} 0)$, проходящей через вакансии, атомы циркония и водорода. Шкала градаций цвета приведена в единицах electron/Bohr ${ }^{3}$.

свидетельствует увеличение по сравнению с системой $\mathrm{Zr}-\mathrm{H}$ (рис. 4,d) числа изолиний, охватывающие эти атомы (рис. 6,c). Детальный анализ распределения электронной плотности системы $\mathrm{Zr}-\mathrm{H}-\mathrm{v}$ показал, что при тетраэдрической координации водорода Т1 и Т2 вблизи вакансии уровень электронной плотности между этим атомом Н и ближайшими к нему атомами $\mathrm{Zr}$ возрастает по сравнению с системой $\mathrm{Zr}-\mathrm{H}$ на 5.0 и $2.4 \%$ соответственно, а при октаэдрической координации на $28.7 \%$. Как известно [24,25], повышение степени ковалентности связей в металле обычно приводит к увеличению хрупкости материала и усилению анизотропии его упругих свойств. Присутствие водорода вблизи вакансии уменьшает область пониженной электронной плотности в ее окрестности, что коррелирует с результатами экспериментов по электронно-позитронной аннигиляции в системах $\mathrm{Ti}-\mathrm{H}-\mathrm{v}$ [26] и $\mathrm{Zr}-\mathrm{H}-\mathrm{v}$ [27], где наблюдается уменьшение времени жизни позитрона в вакансии после растворения водорода.
Как уже было отмечено, образование вакансии приводит к разрыву общего контура изолиний, охватывающих пары атомов циркония 5-13, 7-15 и 8-16, находящихся над и под ней, что означает ослабление связи между этими атомами. Присутствие водорода в тетраэдрическом междоузлии Т2 вблизи вакансии в результате релаксации решетки восстанавливает этот контур для пары атомов циркония 15 и 7, находящихся над и под атомом водорода (рис. $6, b$ ), усиливая связь между этими атомами. В случае систем $\mathrm{Zr}-\mathrm{H}^{\mathrm{T} 1}-v$ (рис. $6, a$ ) и $\mathrm{Zr}-\mathrm{H}^{\mathrm{O}}-v$ (рис. 6, $c$ ) этот контур остается разорванным. По-видимому, это объясняет наибольшую энергию связи водорода с цирконием при его размещении в тетраэдрическом междоузлии Т2.

\section{4. Заключение}

В настоящей работе в рамках теории функционала электронной плотности полнопотенциальным $a b$ initio методом линеаризованных присоединенных плоских 
волн проведена полная релаксация атомной структуры и рассчитано распределение электронной плотности систем $\mathrm{Zr}-\mathrm{v}, \mathrm{Zr}-\mathrm{H}$ и $\mathrm{Zr}-\mathrm{H}-\mathrm{v}$. Показано, что для правильного описания энергетики систем $\mathrm{Zr}-\mathrm{H}$ и $\mathrm{Zr}-\mathrm{H}-\mathrm{v}$ необходимо учитывать не только изменение параметров решетки металла, но и релаксацию положения всех атомов кристалла. Вычислены энергия связи водорода в чистом $\mathrm{Zr}$ и системе $\mathrm{Zr}-v$, энергия образования вакансии в $\mathrm{Zr}$ и системе $\mathrm{Zr}-\mathrm{H}$, а также энергия связи водорода с вакансией в цирконии. Установлено, что наличие вакансий в решетке металла увеличивает энергию связи водорода в цирконии до 53\%, а присутствие водорода уменьшает энергию образования вакансии в цирконии до 14\%. Атому водорода и вакансии энергетически выгодно образовывать комплексы, в которых водород находится в ближайших к вакансии междоузлиях. Вблизи этих комплексов наблюдается повышение степени ковалентности связей в металле. Присутствие водорода вблизи вакансии уменьшает область пониженной электронной плотности в ее окрестности. Это можно наблюдать экспериментально методом электронно-позитронной аннигиляции в виде сокращения времени жизни позитрона в комплексе водород-вакансия.

\section{Список литературы}

[1] S.P. Lynch. In: Stress Corrosion Cracking: Theory and Practice / Eds V.S. Raja, T. Shoji. Woodhead Pub. Ltd., Cambridge. (2011). P. 90-130.

[2] O. Lopatina, L. Svyatkin, Y. Koroteev, I. Chernov. Adv. Mater. Res. 1084, 241 (2015).

[3] J. Č́̌́̌̌ek, I. Procházka, F. Bečvář, R. Kužel, M. Cieslar, G. Brauer, W. Anwand, R. Kirchheim, A. Pundt. Phys. Rev. B 69, 224106 (2004).

[4] J. Cizek, I. Prochazka, S. Danis, M. Cieslar, G. Brauer, W. Anwand, R. Kirchheim, A. Pundt. J. Alloy Comp. 446-447, 479 (2007).

[5] O. Vekilova, D. Bazhanov, S. Simak, I. Abrikosov. Phys. Rev. B 80, 024101 (2009).

[6] M.P. Benediktsson, K.K.G. Mýrdal, P. Maurya, A. Pedersen. J. Phys.: Condens. Matter. 25, 375401 (2013).

[7] W. Xing, X.-Q. Chen, Q. Xie, G. Lu, D. Li, Y. Li. Int. J. Hydrogen Energ. 39, 11321 (2014).

[8] D. Connetable, J. Huez, E. Andrieu, C. Mijoule. J. Phys.: Condens. Matter 23, 405401 (2011).

[9] C. Varvenne, O. Mackain, E. Clouet. Acta Mater. 102, 56 (2016).

[10] A. Zielinski, S. Sobieszczyk. Int. J. Hydrogen Energ. 36, 8619 (2011).

[11] S. Blügel, G. Bihlmayer. Comp. Nanosci. 31, 85 (2006).

[12] http://www.flapw.de

[13] J.P. Perdew, K. Burke, E. Matthias. Phys. Rev. Lett. 77, 3865 (1996).

[14] E.S. Fisher, C.J. Renken. Phys. Rev. 135, A482 (1964).

[15] F.Wang, H.R. Gong. Int. J. Hydrogen Energ. 37, 12393 (2012).

[16] C. Domain, R. Besson, A. Legris. Acta Mater. 50, 3513 (2002).

[17] E. Smith. J. Mater Sci. 30, 5910 (1995).
[18] P. Narang, G. Paul, K. Taylor. J. Less Common Met. 56, 125 (1977).

[19] H. Wenzl. Int. Met. Rev. 27, 140 (1982).

[20] C. Varvenne, O. Mackain, E. Clouet. Acta Mater. 78, 65 (2014).

[21] G.M. Hood, R.J. Schultz, J.A. Jackman. J. Nucl. Mater. 126, 79 (1984).

[22] G.M. Hood. J. Nucl. Mater. 139, 179 (1986).

[23] C. Zhang, A. Alavi. J. Am. Chem. Soc. 127, 9808 (2005).

[24] П.И. Полухин, С.С. Горелик, В.К. Воронцов. Физические основы пластической деформации. Металлургия, М. (1982). $584 \mathrm{c.}$

[25] Л.Д. Соколов, В.А. Скуднов, В.М. Соленов. Механические свойства редких металлов / Под. ред. Л.Д. Соколова. Металлургия, М. (1972). 287 с.

[26] R.S. Laptev, A.M. Lider, Y.S. Bordulev, V.N. Kudiyarov, G.V. Garanin. J. Alloy Comp. 645, 193 (2015).

[27] R.S. Laptev, A.M. Lider, Y.S. Bordulev, V.N. Kudiyarov, D.V. Gvozdyakov. Key Eng. Mater. 683, 256 (2016). 